\title{
Comparação de soluções extratoras de ferro e manganês em solos da Amazônia(1)
}

\author{
Maria do Rosário Lobato Rodrigues ${ }^{(2)}$, Eurípedes Malavolta( ${ }^{(3)}$ e Adônis Moreira(4)
}

\begin{abstract}
Resumo - O objetivo deste trabalho foi comparar soluções extratoras (Mehlich 1, Mehlich 3, DTPA-TEA) de ferro e manganês em solos representativos da Região Amazônica. Foram determinadas as correlações desses micronutrientes nos solos com os teores e conteúdos na matéria seca da parte aérea de plantas de arroz de três cultivos sucessivos. Aplicou-se a técnica do diagnóstico por subtração, em delineamento em blocos casualizados com parcelas subdivididas. Foram utilizados os solos Podzol, Podzólico Amarelo, Podzólico Vermelho-Amarelo, Latossolo Amarelo, Latossolo Húmico e Aluvial, sob oito tratamentos: controle, completo e com omissão de um dos micronutrientes $\mathrm{B}, \mathrm{Cu}, \mathrm{Fe}, \mathrm{Mn}, \mathrm{Mo}$ e Zn. Os micronutrientes e a calagem foram aplicados somente antes do primeiro cultivo. A primeira colheita foi realizada aos 58 dias, a segunda aos 68 e a terceira aos 70 dias após a emergência das plântulas. A solução extratora Mehlich 3 apresentou a maior correlação com o teor de micronutrientes na planta. O melhor coeficiente de determinação foi observado entre as soluções Mehlich 1 e Mehlich 3 quanto aos teores de Fe extraídos dos solos Podzol, Aluvial e Podzólico Vermelho-Amarelo. Com relação ao Mn trocável, os três extratores mostraram-se eficientes na determinação do elemento nos diferentes solos, apresentando coeficientes de determinação significativos entre si.
\end{abstract}

Termos para indexação: análise de solo, métodos de análise, metais pesados, cultivo, arroz.

\section{Comparison of extracting solution for iron and manganese in soils of the Amazon Region}

\begin{abstract}
The aim of this work was to compare extracting solutions (Mehlich 1, Mehlich 3, DTPA-TEA) for iron and manganese in soils of the Amazon Region. The correlations between Fe and $\mathrm{Mn}$ in soils and their contents in the rice plants were determined. The technique of diagnosis by subtractions was used, in a randomized block design with split plots. The soil types were Podzols, Yellow Podzolic, Red-Yellow Podzolic, Yellow Latosol, Humic Latosol, and Alluvial under eight treatments: control, complete with all micronutrients, and omitting one by one of the following micronutrients B, $\mathrm{Cu}, \mathrm{Fe}, \mathrm{Mn}, \mathrm{Mo}$ and $\mathrm{Zn}$. Micronutrients and lime were applied before the first crop. Plants were harvested at 58, 68 and 70 days after emergence. Considering all soils, Mehlich 3 provided the highest correlation coefficients with the content of micronutrients in the plants. The best coefficients of determination were observed with Mehlich 1 and 3 solutions with respect to available Fe in the Podzols, Alluvial and Red-Yellow Podzolic soils. The several extracting solutions were equally efficient for exchangeable Mn determination, and presented significant determination among each other.
\end{abstract}

Index terms: soil testing, analytical methods, heavy metals, cultivation, rice.

(1) Aceito para publicação em 16 de março de 2000. Parcialmente financiado pela FAPESP. Extraído da tese de doutorado apresentada pelo primeiro autor à Escola Superior de Agricultura Luiz de Queiroz (ESALQ) em 1998.

(2) Embrapa-Centro de Pesquisa Agroflorestal da Amazônia Ocidental (CPAA), Caixa Postal 319, CEP 69011-970 Manaus, AM. E-mail: ayres@amazonet.com.br

(3) Universidade de São Paulo, Centro de Energia Nuclear na Agricultura, Caixa Postal 96, CEP 13400-970 Piracicaba, SP. Bolsista do CNPq. E-mail: mala@ cena.usp.br

(4) Embrapa-CPAA. Bolsista do CNPq.

E-mail: adonis@cpaa.embrapa.br

\section{Introdução}

Diversos trabalhos (Camargo et al., 1982; Pavan \& Miyazawa, 1984; Sims, 1985) têm demonstrado que os métodos de análise de solos para micronutrientes ainda não apresentam a segurança desejada, sendo necessários novos estudos para que se definam os métodos de análise de solo confiáveis, para possibilitar uma eficiente diagnose de deficiência nutricional. Nesse contexto, somente após a definição dos métodos é que as análises de micronutrientes 
deverão ocupar lugar de destaque nos programas regionais de controle de qualidade dos laboratórios de análise de solo e tecido de plantas.

Segundo Raij \& Bataglia (1988), as técnicas mais comumente empregadas na seleção de soluções extratoras consistem no cultivo de plantas em diferentes solos com teores variados de nutrientes, ou por meio da variação artificial destes com aplicação de diferentes doses dos elementos, correlacionando-os com o teor no solo e com o teor foliar das amostras estudadas. Na escolha do melhor, é feito o cálculo de correlação simples entre a matéria seca produzida, o teor do elemento e o seu conteúdo na planta com os teores do solo extraídos pelas diferentes soluções; este último parâmetro é admitido como o mais adequado, uma vez que a extração feita pela planta é o melhor índice dos nutrientes no solo (Hauser, 1973).

De acordo com Lindsay \& Cox (1985), os níveis de Fe e de Mn na solução do solo são geralmente muito baixos, o mesmo ocorrendo com as quantidades adsorvidas nos sítios de troca. Por este motivo, a maioria das soluções extratoras usadas na extração do Fe e Mn do solo contêm ácidos, bases, complexantes ou quelatos que também dissolvem parte dos sólidos na fase lábil. No caso dos solos cauliníticos altamente intemperizados e ácidos, os mesmos autores observaram que a utilização de uma solução extratora mais forte, tamponada a valores mais baixos de $\mathrm{pH}$, projetada para simular o ambiente das plantas que se desenvolvem em solos ácidos, seria o mais recomendável.

As soluções extratoras DTPA-TEA e Mehlich $1\left(0,1 \mathrm{~mol} \mathrm{~L}^{-1} \mathrm{de} \mathrm{HCl}+0,0125 \mathrm{~mol} \mathrm{~L}^{-1}\right.$ de $\mathrm{H}_{2} \mathrm{SO}_{4}$ ) são as mais empregadas atualmente pelos laboratórios de análise (Raij, 1991; Embrapa, 1997), porém, poucos estudos mostram correlações entre Fe ou Mn no solo com Fe e Mn na planta. Sabe-se que existe, atualmente, uma tendência mundial de uso de soluções multinutrientes, que extraem diversos elementos simultaneamente, independentemente do tipo de solo. Entre estas, se encaixa a solução Mehlich 3 $\left(0,2 \mathrm{~mol} \mathrm{~L}^{-1}\right.$ de $\mathrm{CH}_{3} \mathrm{COOH}+0,25 \mathrm{~mol} \mathrm{~L}^{-1} \mathrm{de}$ $\mathrm{NH}_{4} \mathrm{NO}_{3}+0,015 \mathrm{~mol} \mathrm{~L}^{-1}$ de $\mathrm{NH}_{4} \mathrm{~F}+0,001 \mathrm{~mol} \mathrm{~L}^{-1}$ de EDTA ácido), que, segundo Tran et al. (1990), pode ser um dos procedimentos mais apropriados para análise de solo em laboratório, por apresentar maior correlação entre nutriente-solo x nutrienteplanta do que o extrator Mehlich 1.

O objetivo deste trabalho foi comparar soluções extratoras (Mehlich 1 e Mehlich 3, DTPA-TEA $\mathrm{pH}$ 7,3) de Fe e Mn em solos representativos da Região Amazônica, através de relações entre os teores desses micronutrientes nos solos e seus teores e conteúdos na planta do arroz.

\section{Material e Métodos}

O experimento foi conduzido em casa de vegetação do Laboratório de Nutrição Mineral de Plantas do Centro de Energia Nuclear na Agricultura (CENA/USP), Piracicaba, SP, entre agosto de 1996 e setembro de 1997. Foram realizados, nesse período, três cultivos sucessivos de arroz, variedade IAC-165, em dez amostras da camada arável $(0-20 \mathrm{~cm})$ de solos representativos da Região Amazônica (Podzol - P, Podzólico Amarelo - PA, Podzólico Vermelho-Amarelo - PV, Latossolo Amarelo - LA, Latossolo Húmico - LH e Aluvial - AL). As amostras foram destorroadas, secadas ao ar e passadas em peneiras com malha de $4 \mathrm{~mm}$ de abertura (TFSA). Foram usados vasos de plástico com capacidade para $500 \mathrm{~mL}$, com fundo perfurado e dotados de coletores para drenagem. Utilizaramse $300 \mathrm{~g}$ de solo e $0,16 \mathrm{dm}^{3}$ de quartzo moído, previamente lavado, sendo que $0,07 \mathrm{dm}^{3}$ desse material foi colocado no fundo do vaso e $0,09 \mathrm{dm}^{3}$ sobre o solo, com o objetivo de melhorar a drenagem e evitar a formação de crostas.

Utilizou-se o diagnóstico por subtração, descrito por Malavolta et al. (1993), adaptado à técnica de Sillanpää (1982), a qual foi empregada no estudo mundial de disponibilidade de micronutrientes. $\mathrm{O}$ delineamento experimental foi em blocos casualizados com parcelas subdivididas, com três repetições. Na casualização, fixaram-se, nas parcelas, as classes de solos, e estes, agrupados em: dois Podzóis álicos, dois Podzólicos Amarelos álicos, um Podzólico Vermelho-Amarelo álico, três Latossolos Amarelos, um Latossolo Húmico antropogênico e um Aluvial eutrófico; foram sorteados nas subparcelas os tratamentos: uma testemunha absoluta (solo original, sem adição de calcário e fertilizantes), um completo (contendo todos macro e micronutrientes), e outros seis tratamentos formados a partir do completo pela omissão de um dos micronutrientes $\mathrm{B}, \mathrm{Cu}, \mathrm{Fe}, \mathrm{Mn}$, Mo e $\mathrm{Zn}$.

$\mathrm{Na}$ correção do solo, elevou-se a saturação por bases a $50 \%$, usando corretivo de acidez na relação de Ca: $\mathrm{Mg}$ 3:1. O P, 1/3 do K, e os micronutrientes (Tabela 1) foram adicionados às amostras de solo junto com a calagem. 
As amostras de solo foram incubadas por um período de 30 dias, com umidade próxima de $70 \%$ do valor total de poros (VTP).

Aplicou-se 1/3 de $\mathrm{N}$ no plantio e os $2 / 3$ restantes desse elemento e de $\mathrm{K}$ foram parcelados em duas aplicações realizadas aos 28 e 40 dias após a germinação (DAG). A umidade do solo foi mantida em torno de $60 \%$ a $70 \%$ do VTP, durante todo o período de cultivo, através de reposições diárias com água destilada. No primeiro cultivo, deixaram-se oito plantas por vaso e o corte do material foi realizado aos 58 DAG. No segundo cultivo, efetuou-se apenas uma adubação básica de manutenção, na forma de solução, com fosfato monoamônio $\left(\mathrm{NH}_{4} \mathrm{H}_{2} \mathrm{PO}_{4}\right)$, fornecendo $100 \mathrm{mg}$ de $\mathrm{P}$ e $44 \mathrm{mg}$ de $\mathrm{N}$ para o Aluvial (AL) e o Latossolo Húmico andropogênico (LH). A diferença com relação ao N para o AL e o LH foi completada com a aplicação de $22 \mathrm{mg}$ de $\mathrm{N}$ na forma de $\mathrm{NH}_{4} \mathrm{NO}_{3}$. Adicionaramse, também, na forma de solução, $50 \mathrm{mg}$ de $\mathrm{K}\left(\mathrm{K}_{2} \mathrm{SO}_{4}\right)$. Durante o desenvolvimento das plantas, realizaram-se mais três aplicações de $\mathrm{N}$ e K: na primeira, aplicaram-se $50 \mathrm{mg}$ de $\mathrm{K}\left(\mathrm{K}_{2} \mathrm{SO}_{4}\right)$ e $50 \mathrm{mg}$ de $\mathrm{N}\left(\mathrm{NH}_{4} \mathrm{NO}_{3}\right)$; na segunda e na terceira, aplicaram-se, em cada uma, $25 \mathrm{mg}$ de $\mathrm{N}$ $\left(\left(\mathrm{NH}_{2}\right)_{2} \mathrm{CO}\right)$ e $25 \mathrm{mg}$ de $\mathrm{K}\left(\mathrm{K}_{2} \mathrm{SO}_{4}\right)$. Foram mantidas 10 plantas por vaso, e a colheita foi realizada aos 68 DAG. No terceiro cultivo, procedeu-se como no segundo, com exceção da adição dos macronutrientes, em que as doses $\left(\mathrm{mg} \mathrm{dm}^{-3}\right)$ no plantio foram: $\mathrm{N}=50\left(\left(\mathrm{NH}_{2}\right)_{2} \mathrm{CO}\right)$, $\mathrm{P}=100\left(\mathrm{NH}_{4} \mathrm{H}_{2} \mathrm{PO}_{4}\right), \mathrm{K}=50\left(\mathrm{~K}_{2} \mathrm{SO}_{4}\right)$. Durante o desen- volvimento das plantas realizaram-se mais quatro aplicações de $25 \mathrm{mg} \mathrm{dm}^{-3}$ cada, para $\mathrm{N}\left(\left(\mathrm{NH}_{2}\right)_{2} \mathrm{CO}\right)$ e para $\mathrm{K}\left(\mathrm{K}_{2} \mathrm{SO}_{4}\right)$. Foram mantidas 17 plantas por vaso, e a colheita foi realizada aos $70 \mathrm{DAG}$.

Nos três cultivos, a parte aérea do arroz foi cortada rente ao solo, lavada com água destilada, acondicionada em sacos de papel e levada para secar em estufa com ventilação forçada e com temperatura média de $65^{\circ} \mathrm{C}$, em que permaneceu até atingir o peso constante. Depois de obtido o peso da matéria seca produzida, o material foi moído e acondicionado em sacos de papel. As determinações dos teores de $\mathrm{Fe}$ e Mn contidos na matéria seca do arroz obedeceram aos métodos descritos por Malavolta et al. (1997).

As amostras de solo foram coletadas após a incubação do solo, antes da realização do primeiro cultivo e após cada cultivo. Essas foram postas para secar dentro da casa de vegetação, a uma temperatura média controlada de $29^{\circ} \mathrm{C}$. O tempo entre a secagem e determinação variou em razão da coleta do solo, que foi feita entre 5 e 10 dias após a última. $\mathrm{O}$ pH em solução $0,01 \mathrm{~mol} \mathrm{~L}^{-1} \mathrm{de} \mathrm{CaCl}_{2}$, de cada solo após a incubação (exceto a testemunha) ficaram nas seguintes faixas: $\mathrm{P}=5,00-6,06, \mathrm{PA}=5,03-7,23$, $\mathrm{PV}=7,22-7,37, \mathrm{LA}=4,29-7,31, \mathrm{LH}=5,39-5,79$, $\mathrm{AL}=4,47-4,74$. O Fe e o Mn do solo foram extraídos pelas seguintes soluções : a) Mehlich $1\left(\mathrm{HCl} 0,05 \mathrm{~mol} \mathrm{~L}^{-1}\right.$ $+\mathrm{H}_{2} \mathrm{SO}_{4} 0,025 \mathrm{~mol} \mathrm{~L}^{-1}$ ) na relação solo:solução 1:5, com 5 minutos de agitação (Mehlich, 1978), modificado por Embrapa (1997); b) Mehlich 3 ( $\mathrm{CH}_{3} \mathrm{COOH} 0,2 \mathrm{~mol} \mathrm{~L}^{-1}$;

Tabela 1. Composição química das soluções nutritivas utilizadas no primeiro cultivo ${ }^{(1)}$.

\begin{tabular}{|c|c|c|c|c|c|c|c|c|c|}
\hline \multirow[t]{2}{*}{ Fonte de nutriente } & \multirow{2}{*}{$\begin{array}{l}\text { Nutriente } \\
\left(\mathrm{mg} \mathrm{dm}^{-3}\right)\end{array}$} & \multicolumn{8}{|c|}{ Tratamento $^{(2)}$} \\
\hline & & $\mathrm{T}$ & $\mathrm{C}$ & $\mathrm{C}-\mathrm{B}$ & $\mathrm{C}-\mathrm{Cu}$ & $\mathrm{C}-\mathrm{Fe}$ & $\mathrm{C}-\mathrm{Mn}$ & C-Mo & $\mathrm{C}-\mathrm{Zn}$ \\
\hline & & \multicolumn{8}{|c|}{ - Volume adicionado (mL/vaso) - } \\
\hline$\left(\mathrm{NH}_{2}\right)_{2} \mathrm{CO}$ & $\mathrm{N}(125)$ & 0 & 10 & 10 & 10 & 10 & 10 & 10 & 10 \\
\hline \multirow[t]{2}{*}{$\mathrm{Ca}\left(\mathrm{H}_{2} \mathrm{PO}_{4}\right)_{2} \cdot \mathrm{H}_{2} \mathrm{O}$} & $\mathrm{P}(200)$ & 0 & 10 & 10 & 10 & 10 & 10 & 10 & 10 \\
\hline & $\mathrm{Ca}(129)$ & - & - & - & - & - & - & - & - \\
\hline \multirow[t]{2}{*}{$\mathrm{K}_{2} \mathrm{SO}_{4}$} & K (125) & 0 & 10 & 10 & 10 & 10 & 10 & 10 & 10 \\
\hline & $S(51)$ & - & - & - & - & - & - & - & - \\
\hline $\mathrm{H}_{3} \mathrm{BO}_{3}$ & B $(0,5)$ & 0 & 10 & 0 & 10 & 10 & 10 & 10 & 10 \\
\hline $\mathrm{CuSO}_{4} .5 \mathrm{H}_{2} \mathrm{O}$ & $\mathrm{Cu}(2,0)$ & 0 & 10 & 10 & 0 & 10 & 10 & 10 & 10 \\
\hline Fe-EDTA & $\mathrm{Fe}(5,0)$ & - & - & - & - & - & - & - & - \\
\hline $\mathrm{MnCl}_{2} \cdot 4 \mathrm{H}_{2} \mathrm{O}$ & $\operatorname{Mn}(5,0)$ & 0 & 10 & 10 & 10 & 10 & 0 & 10 & 10 \\
\hline $\mathrm{MoO}_{3}$ & Mo $(0,1)$ & 0 & 10 & 10 & 10 & 10 & 10 & 0 & 10 \\
\hline $\mathrm{ZnSO}_{4} \cdot 7 \mathrm{H}_{2} \mathrm{O}$ & $\mathrm{Zn}(2,0)$ & 0 & 10 & 10 & 10 & 10 & 10 & 10 & 0 \\
\hline
\end{tabular}

(1) Nas amostras dos solos Aluvial e Latossolo Húmico, utilizou-se metade da dose de P, Cu, Mn e Zn; nas amostras do Latossolo Húmico, utilizou-se metade da dose de B; o volume da solução de Fe-EDTA (mL/vaso) foi adicionado com base no volume dos solos $\left(\mathrm{mg} \mathrm{dm}^{-3}\right)$ : Podzol = 2,1; Podzólico Amarelo e Latossolo Amarelo = 2,9; Latossolo Húmico, Aluvial e Podzólico Vermelho = 3,0. (2) T: testemunha; C: completo ( B, Cu, Fe, Mn, Mo e Zn); C-B: completo sem B; C-Cu: completo sem Cu; C-Fe: completo sem Fe; C-Mn: completo sem Mn; C-Mo: completo sem Mo; C-Zn: completo sem Zn. 
$\mathrm{NH}_{4} \mathrm{NO}_{3} 0,25 \mathrm{~mol} \mathrm{~L}^{-1} ; \mathrm{NH}_{4} \mathrm{~F} 0,015 \mathrm{~mol} \mathrm{~L}^{-1} ; \mathrm{HNO}_{3}$ $0,013 \mathrm{~mol} \mathrm{~L}^{-1}$ e EDTA (ácido etileno diamino tetraacético $0,001 \mathrm{~mol} \mathrm{~L}^{-1}, \mathrm{pH} 2,5$ ) na relação solo:solução 1:10, com 5 minutos de agitação (Mehlich, 1984); c) DTPA-TEA (ácido dietileno triamino pentaacético-trietanolamina) tamponado a pH 7,3, na relação solo:solução de 1:2, com duas horas de agitação (Lindsay \& Norvell, 1978). $\mathrm{Na}$ determinação do $\mathrm{Fe}$ e $\mathrm{Mn}$ utilizou-se o espectrofotômetro de absorção atômica.

Os resultados foram submetidos a análise de variância (teste F), correlação entre os teores obtidos com as soluções extratoras e regressão linear simples, a 5\% de probabilidade (Pimentel-Gomes, 1990), entre os teores e conteúdos de Fe ou de Mn na matéria seca da parte aérea e os seus teores no solo, obtidos com as diferentes soluções extratoras.

\section{Resultados e Discussão}

Houve grande variabilidade dos teores de Fe e de Mn existentes nos diferentes tipos de solos, obtidos pelas soluções extratoras (Tabela 2). Os teores máximos e mínimos de $\mathrm{Fe}$ obtidos pelas soluções Mehlich 1 e DTPA-TEA foram semelhantes e menores do que aqueles obtidos pela solução Mehlich 3 . No primeiro cultivo, os coeficientes de correlação entre os teores de $\mathrm{Fe}$ no solo foram significativos somente com o conteúdo na planta (Tabela 3), no entanto, nos cultivos subseqüentes, os coeficientes mostraram significância entre as correlações feitas com o teor e conteúdo de Fe na matéria seca. Esse resultado contraria uma expectativa inicial, visto que Bataglia \& Raij (1989) não encontraram correlações significativas entre os teores desses nutrientes no solo e na planta usando soluções ácidas. A solução
Mehlich 3 mostrou maior capacidade de extrair $\mathrm{Fe}$, possivelmente pela sua capacidade ácida e pela presença do EDTA. No primeiro cultivo, as concentrações médias desse elemento extraídas pela solução Mehlich 3 foram 1,8 e 1,9 vezes maior do que aquelas extraídas pela solução Mehlich 1 e DTPA-TEA, respectivamente. No terceiro cultivo, essa diferença acentuou-se, passando para 2,0 e 2,6 vezes maior do que das soluções Mehlich $1 \mathrm{e}$ DTPA-TEA, respectivamente (Tabela 2). A superioridade da solução Mehlich 3 na capacidade de extrair Fe também foi comprovada por Alva (1992), em estudo comparativo entre diversas soluções extratoras em 340 amostras de solos do estado da Flórida, EUA, cultivados com citros.

Com relação aos teores de Mn no solo, as soluções DTPA-TEA e Mehlich 1 foram menos eficientes que a solução Mehlich 3, apresentando, na média, as menores capacidades de extração e as menores correlações com os teores e conteúdo presente na matéria seca da planta (Tabelas 2 e 3). Mehlich (1984), em seu trabalho no desenvolvimento da solução Mehlich 3, relata que a incorporação do EDTA nas soluções extratoras, tais como DTPA-TEA e Mehlich 3, tem a finalidade de melhorar a extração de micronutrientes do solo, o que foi confirmado no presente trabalho (Tabela 3).

Com relação às soluções extratoras Mehlich $1 \mathrm{e}$ DTPA-TEA, exceto no primeiro cultivo, em que a solução Mehlich 1 apresentou maior coeficiente de correlação com o teor de Mn na matéria seca (Tabela 3), os resultados encontrados no presente trabalho concordam com os obtidos por Abreu et al. (1996),

Tabela 2. Teores de ferro e manganês nos solos determinados com três soluções extratoras.

\begin{tabular}{|c|c|c|c|c|c|c|c|}
\hline \multirow[t]{2}{*}{ Micronutriente } & \multirow{2}{*}{$\begin{array}{l}\text { Solução } \\
\text { extratora }\end{array}$} & \multicolumn{3}{|c|}{ Antes do $1^{\circ}$ cultivo } & \multicolumn{3}{|c|}{ Após o $3^{\circ}$ cultivo } \\
\hline & & Mínimo & Máximo & Média & Mínimo & Máximo & Média \\
\hline & & ------ & 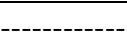 & --- (mg & 3) -- & & \\
\hline \multirow[t]{3}{*}{$\mathrm{Fe}$} & Mehlich 1 & 13,62 & 758,78 & 261,44 & 10,90 & 239,29 & 116,97 \\
\hline & Mehlich 3 & 104,84 & 944,12 & 461,24 & 94,24 & 555,64 & 309,03 \\
\hline & DTPA-TEA & 20,48 & 741,41 & 242,46 & 14,62 & 348,16 & 153,55 \\
\hline \multirow[t]{3}{*}{$\mathrm{Mn}$} & Mehlich 1 & 4,02 & 251,11 & 74,11 & 0,48 & 64,22 & 18,12 \\
\hline & Mehlich 3 & 4,03 & 142,57 & 27,78 & 0,63 & 81,41 & 19,55 \\
\hline & DTPA-TEA & 1,47 & 112,60 & 33,74 & 0,14 & 59,27 & 13,84 \\
\hline
\end{tabular}


que ao estudar a eficiência de soluções extratoras na determinação do Mn disponível observou que a solução DTPA-TEA foi a que apresentou o maior coeficiente de correlação quando comparada à de Mehlich 1.

Tabela 3. Coeficientes de correlação simples entre os teores de ferro e manganês do solo, determinados pelas soluções extratoras, e seus teores e conteúdos na matéria seca da parte aérea de plantas de arroz. Média de todos os solos.

\begin{tabular}{clccc}
\hline \multirow{2}{*}{ Micronutriente } & Solução & \multicolumn{3}{c}{ Cultivo } \\
\cline { 3 - 5 } & extratora & $1^{\text {o }}$ & $2^{-}$ & $3^{\circ}$ \\
\hline & & \multicolumn{3}{c}{ Teor } \\
$\mathrm{Fe}$ & Mehlich 1 & $0,128^{\mathrm{ns}}$ & $0,572^{*}$ & $0,540^{*}$ \\
$\mathrm{Fe}$ & Mehlich 3 & $0,327^{\mathrm{ns}}$ & $0,566^{*}$ & $0,580^{*}$ \\
$\mathrm{Fe}$ & DTPA-TEA & $0,140^{\mathrm{ns}}$ & $0,502^{*}$ & $0,613^{*}$ \\
$\mathrm{Mn}$ & Mehlich 1 & $0,760^{*}$ & $0,834^{*}$ & $0,681^{*}$ \\
$\mathrm{Mn}$ & Mehlich 3 & $0,778^{*}$ & $0,907^{*}$ & $0,822^{*}$ \\
$\mathrm{Mn}$ & DTPA-TEA & $0,651^{*}$ & $0,873^{*}$ & $0,699^{*}$ \\
\hline & & \multicolumn{3}{c}{ Conteúdo } \\
$\mathrm{Fe}$ & Mehlich 1 & $0,439^{*}$ & $0,692^{*}$ & $0,458^{*}$ \\
$\mathrm{Fe}$ & Mehlich 3 & $0,620^{*}$ & $0,592^{*}$ & $0,413^{*}$ \\
$\mathrm{Fe}$ & DTPA-TEA & $0,512^{*}$ & $0,677^{*}$ & $0,580^{*}$ \\
$\mathrm{Mn}$ & Mehlich 1 & $0,797^{*}$ & $0,741^{*}$ & $0,617^{*}$ \\
$\mathrm{Mn}$ & Mehlich 3 & $0,825^{*}$ & $0,862^{*}$ & $0,773^{*}$ \\
$\mathrm{Mn}$ & DTPA-TEA & $0,662^{*}$ & $0,737^{*}$ & $0,614^{*}$ \\
\hline $\mathrm{ns} \mathrm{Não-significativo}$ & $*$ Significativo a $5 \%$ de probabilidade, pelo teste F
\end{tabular}

ns Não-significativo. * Significativo a 5\% de probabilidade, pelo teste F.
A Tabela 4 mostra as correlações entre as soluções Mehlich 1, Mehlich 3 e DTPA-TEA com os seus coeficientes em relação aos teores de Fe e Mn nos solos agrupados em seis tipos dominantes.

Observa-se que com os teores de $\mathrm{Mn}$, apesar das variações nos teores (Tabela 2), as soluções extratoras apresentaram entre si, elevado grau de correlação, independentemente do tipo de solo (Tabela 4). Essa similaridade é confirmada pelas correlações significativas entre os teores de Mn obtidos em cada cultivo com o seu teor ou conteúdo na planta (Tabela 3). Tais resultados são corroborados por diversos trabalhos realizados em outras condições edafoclimáticas (Sims, 1985; Bataglia \& Raij, 1989; Wendt, 1995). Quando foram considerados todos os solos, as correlações entre os teores de Mn determinados pelas soluções extratoras foram significativas (Tabela 4).

Os valores de Fe extraídos pelas três soluções foram semelhantes aos obtidos por Bataglia \& Raij (1989), com as soluções Mehlich 1 e DTPATEA e por Wendt (1995), com as soluções Mehlich 3 e DTPA-TEA. Os solos Podzol, Aluvial e Podzólico Vermelho-Amarelo apresentaram maior correlação apenas entre as soluções Mehlich 1 e Mehlich 3, com coeficientes significativos (Tabela 4). Quando foram considerados todos os solos, as três soluções extratoras apresentaram a mesma tendência, com coeficientes de regressão significativos a $5 \%$ de probabilidade (Tabela 4).

Tabela 4. Equações de regressão e coeficientes de determinação entre os teores de Fe e de Mn determinados com as soluções extratoras, nos solos amostrados antes da semeadura e depois de cada cultivo de arroz, em todos os tratamentos.

\begin{tabular}{|c|c|c|}
\hline \multirow[t]{2}{*}{ Solução extratora } & \multicolumn{2}{|c|}{ Nutrientes } \\
\hline & Ferro & Manganês \\
\hline & \multicolumn{2}{|c|}{ Aluvial } \\
\hline Mehlich 1 vs. Mehlich 3 & $\begin{array}{l}Y=174,16+0,538 * * X \\
R^{2}=0,662\end{array}$ & $\begin{array}{l}Y=0,307+0,865 * * X \\
R^{2}=0,929\end{array}$ \\
\hline Mehlich 1 vs. DTPA-TEA & $\begin{array}{l}Y=128,50+0,011^{\mathrm{ns}} \mathrm{X} \\
\mathrm{R}^{2}=0,002\end{array}$ & $\begin{array}{l}\mathrm{Y}=0,063+0,503 * * \mathrm{X} \\
\mathrm{R}^{2}=0,773\end{array}$ \\
\hline \multirow[t]{2}{*}{ Mehlich 3 vs. DTPA-TEA } & $\begin{array}{l}Y=125,06+0,020^{\text {ns }} X \\
R^{2}=0,003\end{array}$ & $\begin{array}{l}\mathrm{Y}=0,152+0,400 * * \mathrm{X} \\
\mathrm{R}^{2}=0,890\end{array}$ \\
\hline & \multicolumn{2}{|c|}{ Latossolo } \\
\hline Mehlich 1 vs. Mehlich 3 & $\begin{array}{l}Y=205,04+0,297 * X \\
R^{2}=0,466\end{array}$ & $\begin{array}{l}\mathrm{Y}=0,235+0,819 * * \mathrm{X} \\
\mathrm{R}^{2}=0,954\end{array}$ \\
\hline Mehlich 1 vs. DTPA-TEA & $\begin{array}{l}Y=95,528+0,394 * X \\
R^{2}=0,344\end{array}$ & $\begin{array}{l}Y=-0,170+0,539 * * X \\
R^{2}=0,656\end{array}$ \\
\hline Mehlich 3 vs. DTPA-TEA & $\begin{array}{l}Y=-13,767+0,494 * * X \\
R^{2}=0,636\end{array}$ & $\begin{array}{l}Y=-0,295+0,648 * * X \\
R^{2}=0,931\end{array}$ \\
\hline
\end{tabular}


Tabela 4. Continuação

\begin{tabular}{|c|c|c|}
\hline \multirow[t]{2}{*}{ Solução extratora } & \multicolumn{2}{|c|}{ Nutrientes } \\
\hline & Ferro & Manganês \\
\hline \multicolumn{3}{|c|}{ Latossolo Húmico antropogênico } \\
\hline Mehlich 1 vs. Mehlich 3 & $\begin{array}{l}Y=-15,011+14,020 * * X \\
R^{2}=0,586\end{array}$ & $\begin{array}{l}Y=60,674+0,448 * * X \\
R^{2}=0,835\end{array}$ \\
\hline Mehlich 1 vs. DTPA-TEA & $\begin{array}{l}Y=-8,817+4,603 * * X \\
R^{2}=0,562\end{array}$ & $\begin{array}{l}Y=45,499+0,217 * * X \\
R^{2}=0,656\end{array}$ \\
\hline \multirow[t]{2}{*}{ Mehlich 3 vs. DTPA-TEA } & $\begin{array}{l}\mathrm{Y}=8,636+0,263 * * \mathrm{X} \\
\mathrm{R}^{2}=0,597\end{array}$ & $\begin{array}{l}Y=24,978+0,406^{* *} \mathrm{X} \\
\mathrm{R}^{2}=0,551\end{array}$ \\
\hline & \multicolumn{2}{|c|}{ Podzol } \\
\hline Mehlich 1 vs. Mehlich 3 & $\begin{array}{l}\mathrm{Y}=24,514+1,498 * * \mathrm{X} \\
\mathrm{R}^{2}=0,859\end{array}$ & $\begin{array}{l}Y=0,103+1,062 * * X \\
R^{2}=0,968\end{array}$ \\
\hline Mehlich 1 vs. DTPA-TEA & $\begin{array}{l}Y=0,223+0,004^{\mathrm{ns}} \mathrm{X} \\
\mathrm{R}^{2}=0,203\end{array}$ & $\begin{array}{l}\mathrm{Y}=0,136+0,227 * * \mathrm{X} \\
\mathrm{R}^{2}=0,453\end{array}$ \\
\hline \multirow[t]{2}{*}{ Mehlich 3 vs. DTPA-TEA } & $\begin{array}{l}\mathrm{Y}=0,114+0,001^{\mathrm{ns}} \mathrm{X} \\
\mathrm{R}^{2}=0,116\end{array}$ & $\begin{array}{l}\mathrm{Y}=0,129+0,208 * * \mathrm{X} \\
\mathrm{R}^{2}=0,442\end{array}$ \\
\hline & \multicolumn{2}{|c|}{ Podzólico Amarelo } \\
\hline Mehlich 1 vs. Mehlich 3 & $\begin{array}{l}Y=306,36+0,804^{\mathrm{ns}} \mathrm{X} \\
\mathrm{R}^{2}=0,203\end{array}$ & $\begin{array}{l}\mathrm{Y}=0,093+0,943 * * \mathrm{X} \\
\mathrm{R}^{2}=0,954\end{array}$ \\
\hline Mehlich 1 vs. DTPA-TEA & $\begin{array}{l}Y=85,99+0,664^{\mathrm{ns}} \mathrm{X} \\
\mathrm{R}^{2}=0,309\end{array}$ & $\begin{array}{l}\mathrm{Y}=-0,023+0,403 * * \mathrm{X} \\
\mathrm{R}^{2}=0,925\end{array}$ \\
\hline \multirow[t]{3}{*}{ Mehlich 3 vs. DTPA-TEA } & $\mathrm{Y}=-18,405+0,499 * * \mathrm{X}$ & $\mathrm{Y}=-0,036+0,417 * * \mathrm{X}$ \\
\hline & $\mathrm{R}^{2}=0,441$ & $\mathrm{R}^{2}=0,442$ \\
\hline & Podzól & relo \\
\hline Mehlich 1 vs. Mehlich 3 & $\begin{array}{l}\mathrm{Y}=393,78+0,297 * X \\
\mathrm{R}^{2}=0,379\end{array}$ & $\begin{array}{l}\mathrm{Y}=0,349+0,865 * * \mathrm{X} \\
\mathrm{r}=0,942\end{array}$ \\
\hline Mehlich 1 vs. DTPA-TEA & $\begin{array}{l}Y=101,20+0,185^{\mathrm{ns}} \mathrm{X} \\
\mathrm{R}^{2}=0,228\end{array}$ & $\begin{array}{l}Y=-0,233+0,529 * * X \\
R^{2}=0,912\end{array}$ \\
\hline \multirow[t]{2}{*}{ Mehlich 3 vs. DTPA-TEA } & $\mathrm{Y}=101,79+0,094^{\mathrm{ns}} \mathrm{X}$ & $\mathrm{Y}=-0,394+0,719 * * \mathrm{X}$ \\
\hline & $\mathrm{R}^{2}=0,014$ & $\mathrm{R}^{2}=0,889$ \\
\hline \multirow[b]{2}{*}{ Mehlich 1 vs. Mehlich 3} & & \\
\hline & $\begin{array}{l}Y=177,15+1,0571 * X \\
R^{2}=0,788\end{array}$ & $\begin{array}{l}\mathrm{Y}=5,8439+0,6564 * * \mathrm{X} \\
\mathrm{R}^{2}=0,879\end{array}$ \\
\hline Mehlich 1 vs. DTPA-TEA & $\begin{array}{l}Y=34,717+0,7435 * X \\
R^{2}=0,727\end{array}$ & $\begin{array}{l}\mathrm{Y}=2,752+0,4784 * * \mathrm{X} \\
\mathrm{R}^{2}=0,919\end{array}$ \\
\hline \multirow[t]{2}{*}{ Mehlich 3 vs. DTPA-TEA } & $Y=-72,515+0,658 * X$ & $Y=-0,3254+0,6944 * * X$ \\
\hline & $\mathrm{R}^{2}=0,808$ & $\mathrm{R}^{2}=0,948$ \\
\hline
\end{tabular}

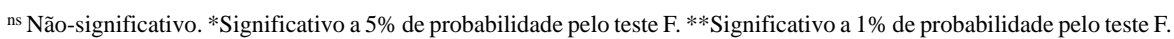

\section{Conclusões}

1. A solução Mehlich 3 extrai mais Fe e Mn que as soluções Mehlich 1 e DTPA-TEA.

2. Os melhores coeficientes de correlação entre os teores de Fe e Mn no solo com o teor e conteúdo na planta são obtidos pela solução Mehlich 3 .

3. As soluções Mehlich 1, Mehlich 3 e DTPA-TEA são mais eficientes na avaliação do Mn na planta em relação ao Fe.

\section{Referências}

ALVA, A. K. Micronutrients status of Florida under citrus production. Communications in Soil Science and Plant Analysis, New York, v. 23, n. 17/20, p. 2493-2510, 1992. ABREU, C. A.; RAIJ, B. van; TANAKA, R. T. Fontes de manganês para soja e seus efeitos na análise do solo. Revista Brasileira de Ciência do Solo, Campinas, v. 20, n. 1, p. 91-97, 1996. 
BATAGLIA, O. C.; RAIJ, B. van. Eficiência de extratores de micronutrientes na análise de solo. Revista Brasileira de Ciência do Solo, Campinas, v. 13, n. 2, p. 205-212, 1989.

CAMARGO, O. A.; VALADARES, J. M. A. S.; DECHEN, A. R. Efeito do pH e da incubação na extração do manganês, zinco, cobre e ferro no solo. Revista Brasileira de Ciência do Solo, Campinas, v. 6, n. 2, p. 83-88, 1982.

EMBRAPA. Centro Nacional de Pesquisas do Solo (Rio de Janeiro, RJ). Manual de métodos de análise do solo. Rio de Janeiro, 1997. 212 p.

HAUSER, G. F. The calibration of soil tests for fertilizer recommendations. Roma : FAO, 1973. 71 p.

LINDSAY, W. L.; COX, F. R. Micronutrients soil testing for tropics. In: VLEK, P. L. G. (Ed.). Micronutrients in tropical food crop production: developments in plant and soil sciences. Dordrecht : M. Nighoff, 1985. v. 14, p. $169-200$.

LINDSAY, W. L.; NORVELL, W. A. Development of a DTPA test for zinc, iron, manganese and copper. Soil Science Society of America Journal, Madison, v. 42, n. 2, p. 421-428, 1978.

MALAVOLTA, E.; MURAOKA, T.; BOARETTO, A. E. Avaliação da fertilidade do solo pela técnica do diagnóstico por subtração. Piracicaba : USP-CENA, 1993. 42 p.

MALAVOLTA, E.; VITTI, G. C.; OLIVEIRA, S. A. Avaliação do estado nutricional das plantas: princípios e aplicações. Piracicaba : Potafos, 1997. 319 p.

MEHLICH, A. Mehlich 3 soil test extractant: a modification of Melich 2 extractant. Communications in Soil Science and Plant Analysis, New York, v. 15, n. 12, p. 1409-1416, 1984.

MEHLICH, A. New extractant for soil test evaluation of phosphorus, potassium, magnesium, calcium, sodium, manganese and zinc. Communications in Soil Science and Plant Analysis, New York, v. 9, n. 2, p. 477-492, 1978.

PAVAN, M. A.; MIYAZAWA, M. Disponibilidade do manganês no solo: dificuldades e problemas na interpretação da análise para fins de fertilidade. Revista Brasileira de Ciência do Solo, Campinas, v. 8, n. 3, p. 285-289, 1984.

PIMENTEL-GOMES, F. Curso de Estatística Experimental. Piracicaba : Nobel, 1990. 468 p.

RAIJ, B. van. Fertilidade do solo e adubação. Piracicaba : Agronômica Ceres/Potafos, 1991. 343 p.

RAIJ, B. van; BATAGLIA, O. C. Análise química do solo para micronutrientes. In: SIMPÓSIO SOBRE MICRONUTRIENTES NA AGRICULTURA, 1. , 1988, Jaboticabal. Anais... Jaboticabal : UNESP, 1988. v. 2, p. $537-569$.

SILLANPÄ̈̈, M. Micronutrients and nutrients status of soils: a global study. Roma : FAO, 1982. 444 p.

SIMS, J. T. A comparison of Mehlich I and Mehlich III extractants of predictors of manganese, copper and zinc availability in four Delaware soils. Communications in Soil Science and Plant Analysis, New York, v. 16, n. 10, p. 1039-1052, 1985.

TRAN, T. S.; GIROUX, M.; GUILBEAUT, J.; AUDESS, P. Evaluation of Mehlich III extractant to estimate the available $\mathrm{P}$ in Quebec soils. Communications in Soil Science and Plant Analysis, New York, v. 21, n. 1, p. 1-28, 1990.

WENDT, J. W. Evaluation of the Mehlich 3 soil extractant for upland Malawi soils. Communications in Soil Science and Plant Analysis, New York, v. 26, n. 5/6, p. 687-702, 1995. 